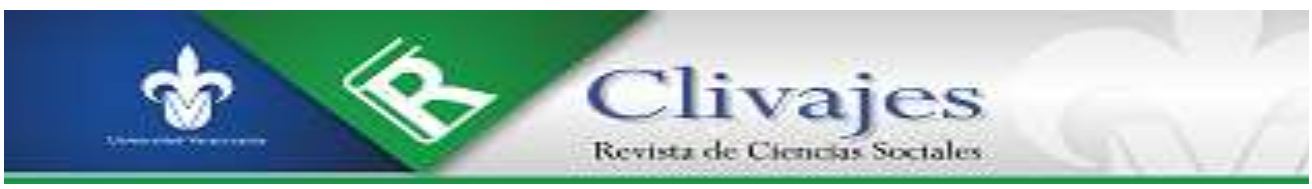

Estefanía Cruz Lera

estefania.1616@,comunidad.unam.mx

Centro de Investigaciones sobre América del Norte, UNAM

INNOVACIÓN SOCIAL Y POLÍTICA DE LOS COLECTIVOS MEXICANOS DE ESTATUS MIXTO

EN ZONAS METROPOLITANAS DE LOS ESTADOS UNIDOS:

BUENAS PRÁCTICAS Y NUEVOS RETOS DURANTE LA COVID-19

DOI: https://doi.org/10.25009/clivajesrcs.i15.2697

Clivajes. Revista de Ciencias Sociales. Año VIII, número 15, enero-junio 2021, pp. 191-213.

https://clivajes.uv.mx/index.php/Clivajes

Instituto de Investigaciones Histórico-Sociales, Universidad Veracruzana

Clivajes. Revista de Ciencias Sociales/ISSN: 2395-9495/IIH-S, UV/Xalapa, Veracruz, México

Recibido: 05/04/2021

Aceptado: 26/04/2021

Dictaminado: $15 / 06 / 2021$

(c) (i) (9)

Clivajes. Revista de Ciencias Sociales (ISSN: 2395-9495), Año VIII, Núm. 15, enero-junio, 2021 Instituto de Investigaciones Histórico-Sociales, Universidad Veracruzana, México 


\title{
INNOVACIÓN SOCIAL Y POLÍTICA DE LOS COLECTIVOS MEXICANOS DE ESTATUS MIXTO EN ZONAS METROPOLITANAS DE LOS ESTADOS UNIDOS: BUENAS PRÁCTICAS Y NUEVOS RETOS DURANTE LA COVID-19
}

\author{
Estefanía Cruz Lera*
}

Resumen

En las grandes urbes de Estados Unidos, los migrantes mexicanos han diversificado sus perfiles dando lugar a comunidades de origen migrante que se caracterizan por su estatus mixto. Mediante la documentación de su trabajo organizacional y su activismo frente a causas locales, en este artículo se identifica dos grandes estrategias que éstos utilizan para enfrentar los retos de sus comunidades: campañas de innovación social y tácticas de incidencia política; la primera, relacionada con actividades de emprendimiento social para mejorar la vida cotidiana de sus miembros y empoderarlos socioeconómicamente; la segunda, asociada al consenso de demandas, la negociación comunitaria de estrategias colectivas, la vinculación con los programas públicos de la ciudad y la construcción de alianzas con actores políticos locales. Con base en el trabajo de campo realizado en zonas metropolitanas de EE.UU., que consistió en observación participante, entrevistas y etnografía digital durante la pandemia, se documentan las buenas prácticas de innovación social e incidencia política de los migrantes y se explican los cambios que enfrentaron a causa de la Covid19.

Palabras clave: Estatus migratorio mixto, Innovación social, Incidencia política, Zonas metropolitanas

\section{SOCIAL AND POLITICAL INNOVATION OF MEXICAN MIXED-STATUS GROUPS IN METROPOLITAN AREAS OF THE UNITED STATES: GOOD PRACTICES AND NEW CHALLENGES DURING COVID-19}

\begin{abstract}
Mexican migrants in large cities of the United States have diversified their profiles, giving rise to communities of migrant origin that are now characterized by their mixed status. From the documentation of their organizational work and their activism in support of their local causes, two great strategies to face the challenges of their communities highlighted. The first type is related to social entrepreneurship to improve the daily lives of its members and empower them socio-economically, these are the social innovation campaigns. The second type is related to the consensus of claims, the community negotiation of collective strategies, the linkage with the city's public programs and the forging of alliances with local political actors; this is what is known as policy incidence. Through fieldwork conducted in US metro areas consisted of participant observation and interviews that was complemented with a digital ethnography during the pandemic, best practices were documented and the changes in activism generated by the Covid-19 are explained.
\end{abstract}

Keywords: Mixed status, Social innovation, Policy incidence, Metropolitan areas

\footnotetext{
* Profesora-investigadora en el Centro de Investigaciones sobre América del Norte de la Universidad Nacional Autónoma de México y miembro del Sistema Nacional de Investigadores, es doctora en Ciencias Políticas, Políticas Públicas y Relaciones Internacionales por la Universidad Autónoma de Barcelona; ha realizado estancias de investigación en ETH-Zúrich (Suiza), Brown University y Georgetown University (Estados Unidos). Actualmente es miembro del Instituto de Investigación Avanzada en Etnicidad, Conflicto y Desigualdad de la Universidad de Brown, de los grupos de trabajo "Latinos, Migración y Diásporas" de LASA y "Liderazgo y Clientelas Políticas” de ALACIP. En 2017 fue reconocida como Investigadora Emergente en Diversidad e Inclusión Social por la alianza Fundación Mellon-CunY.
} 
INNOVATION SOCIALE ET POLITIQUE DES COLLECTIFS MEXICAINS DE STATUT MIXTE EN ZONES METROPOLITAINES DES ÉTATS-UNIS : BONNES PRATIQUES ET NOUVEAUX DEFIS PENDANT LA COVID-19

\section{Résumé}

Dans les grandes métropoles des États-Unis, les migrants mexicains ont diversifié leurs profils en donnant lieu à des communautés d'origine migrante qui se caractérisent par leur statut mixte. On identifie deux grandes stratégies qu'utilisent les migrants pour faire face aux défis de leurs communautés à travers la documentation du travail organisationnel et de leur activisme face à des causes locales : des campagnes d'innovation sociale et des tactiques d'incidence politique ; la première, liée à des activités d'entrepreneuriat social pour améliorer la vie quotidienne de leurs membres et leur donner du pouvoir socioéconomique; la deuxième, associée au consensus de demandes, la négociation communautaire de stratégies collectives, la corrélation avec les programmes publics de la ville et la construction d'alliances avec des acteurs politiques locaux. À partir du travail sur le terrain réalisé dans des zones métropolitaines des États-Unis, à base d'une observation participative, des interviews et de l' ethnographie numérique pendant la pandémie, on a documenté les bonnes pratiques d'innovation sociale et d'incidence politique des migrants et on explique les changements qu'ils ont affronté à cause de la Covid-19.

Mots clés : Statut migratoire mixte, Innovation sociale, Incidence politique, Zones métropolitaines

\section{INTRODUCCIÓN}

Hay dos perspectivas generales para entender la incorporación de los inmigrantes a la vida política local (Martiniello \& Lafleur, 2008). La primera se relaciona con explicaciones basadas en los recursos de los grupos y la segunda implica enfoques contextuales, es decir, en el primer caso, el éxito de los colectivos se basa en las capacidades adquiridas por la masa crítica guiando a los inmigrantes, mientras que la segunda depende de catalizadores como la tolerancia y las herramientas ofrecidas por los contextos de recepción. La principal diferencia entre ambas perspectivas consiste en la variable explicativa que se privilegie al abordar la dinámica en cuestión. En el caso de este estudio, se pone un énfasis en la agencia política de los inmigrantes latinos en Estados Unidos, en la experiencia que tras un siglo de presencia y de activismo en ese país han adquirido.

El objetivo principal de la investigación consiste en reflexionar sobre las estrategias para hacer frente a los desafíos que afectan a las comunidades migrantes mexicanas de estatus mixto que habitan en áreas metropolitanas de Estados Unidos. Se estudian sus innovaciones comunitarias y los procesos de incidencia política, documentando las buenas prácticas y sobre todo analizando cómo la pandemia de la Covid-19 ha afectado la construcción de comunidad mediante el emprendimiento social y la práctica política. Este artículo contribuye a llenar la brecha de investigación que 
persiste en México sobre la actualidad de la evolución comunitaria y la maduración política que están experimentando los grupos de origen mexicano en Estados Unidos.

El término de comunidades migrantes de estatus mixto engloba a los inmigrantes de primera generación, a los residentes permanentes, a los DACAmentados, a los inmigrantes de segunda generación y a los demás estatus migratorios que suelen confluir al interior de las familias y los vecindarios de origen mexicano en Estados Unidos (Enriquez, 2015; Mangual Figueroa, 2012). Al ser unidades sociales más diversas, tienen diferentes ideologías y prioridades cívicas y políticas contrastantes; sin embargo, la concentración geográfica, los vínculos sociales y culturales, así como las interacciones cotidianas, los han obligado a encontrar fuentes de cohesión social y causas locales compartidas. En esta investigación se han documentado las buenas prácticas de incidencia política en sus zonas metropolitanas y también se reflexiona sobre los desafíos que la pandemia global del coronavirus ha dejado para un activismo que ya estaba dejando huella en la vida cívica y política de las urbes estadunidenses.

Este estudio recupera entrevistas semiestructuradas con líderes migrantes y notas de campo de la observación participante de los años de 2016, 2017 y 2018 en Illinois, California y Texas, trabajo de campo que durante 2020 ha sido complementado por una etnografía digital (Côté, 2013; Kosinski et al., 2015). El seguimiento virtual fue posible gracias a la previa identificación de organizaciones transcendentales durante el trabajo de campo presencial. Esta identificación previa permitió localizar a las entidades clave y seguir su activismo en redes. Las organizaciones étnicas han aprovechado la visibilidad, la difusión y el aforo de las redes sociales para escalar el alcance de sus campañas; por eso, ahora es posible y fructífero documentar su activismo a través de etnografías digitales. Así, se logró dar seguimiento a dinámicas de innovación social e influencia política que se venían gestando en las comunidades de estatus mixto y que se tuvieron que reinventar ante la pandemia del Covid-19.

Este artículo se estructura en cinco secciones. La primera nos permite contextualizar el activismo migrante en las zonas metropolitanas de Estados Unidos; aquí se aborda por qué las urbes son suelos fértiles para las iniciativas comunitarias de los migrantes; la segunda explica en qué consisten la innovación social y la incidencia política como marcos analíticos para entender las estrategias de influencia y emprendimiento social de los inmigrantes; la tercera documenta las mejores prácticas de innovación social e incidencia política desarrolladas por las comunidades mixtas de origen mexicano en Estados Unidos; en la cuarta sección se reflexiona sobre cómo la pandemia de Covid-19 trajo consigo un conjunto de barreras a las estrategias para enfrentar el censo y las 
elecciones de 2020, así como nuevos desafíos para paliar los efectos nocivos sobre la salud y la economía de las comunidades de estatus mixto. Finalmente, en la discusión final se hace un balance sobre la agencia política de las comunidades de estatus mixto.

\section{MigRACIÓN Y ZONAS METROPOLITANAS}

Las grandes zonas metropolitanas experimentan de forma diferente a la migración, comparadas con las comunidades rurales o las pequeñas ciudades (Mollenkopf \& Sonenshein, 2009). Las grandes urbes estadunidenses han estado mayormente expuestas a las diferentes oleadas de inmigrantes, son las principales puertas de entrada de la migración en redes, están más acostumbradas a la conformación de enclaves étnicos y, por tanto, son espacios más fructíferos para la innovación social migrante.

Las ciudades son el epicentro de los debates contemporáneos sobre la movilidad humana con cerca del 54\% de la población mundial contemporánea viviendo en ciudades. Además, la mayoría de los inmigrantes contemporáneos a nivel mundial (54\%) están viviendo en ciudades (Price \& Chacko, 2016). Saskia Sassen (1990) explica que una de las principales paradojas de la globalización es que implica un sistema en el que el capital se mantiene como un asunto global, pero el trabajo y su regularización continúan siendo un asunto local. Contexto en el que, además, los inmigrantes se posicionan como actores transnacionales.

De acuerdo con estimaciones del Pew Research Center, seis de cada diez inmigrantes en Estados Unidos se concentran en tan sólo veinte áreas metropolitanas (Passel \& Cohn, 2017). Las ciudades, en particular las de California, Texas, Illinois y Nueva York han sido históricamente la principal puerta de entrada y las geografías primordiales para la conformación de comunidad de los migrantes mexicanos (Schaeffer, 2019).

Los perfiles de los latinos en Estados Unidos se han diversificado, la mayoría ya no corresponde a población indocumentada nacida en el extranjero, el 60\% de los latinos han nacido en Estados Unidos, el 20\% son ciudadanos por naturalización (GonzálezBarrera, 2017). Además, hay 27 millones de latinos que pueden votar en Estados Unidos y hay cada vez más latinos en puestos públicos (Krogstad et al., 2019); algunos de ellos inclusive nacidos en el extranjero, como los congresistas Chuy García, Raúl Ruíz y Salud Carbajal que nacieron en México. Toda esta diversidad nos obliga a repensar las interacciones sociales y políticas de los latinos, al interior y hacia el exterior de este 
colectivo cada vez más diverso en términos de estatus, clase e ideología, pero también cada vez más influyente en el país vecino.

La cadena de eventos en detrimento de los inmigrantes que se han detonado en los últimos años se caracterizó por su criminalización y su cualificación como carga pública para la sociedad estadunidense. Estas ideologías y su materialización en políticas han obligado a las comunidades de estatus mixto a tener que convertirse en entidades creativas. La masa crítica latina apuesta por convertir a sus organizaciones en semilleros de propuestas políticas, las cuales están ancladas en las oportunidades políticas para inmigrantes y minorías que ofrecen las ciudades de asentamiento, y cuyas propuestas, a su vez, sean capaces de vincularse con iniciativas más generales, de tal forma que sean aplaudidas entre el núcleo social dominante.

Numerosos estudios han abordado las interrogantes de por qué las localidades se han posicionado como epicentros de resistencia en contra de la política migratoria antiinmigrante (Cruz Lera, 2019; Varsanyi et al., 2011). Los representantes latinos se han convertido en campeones defendiendo a sus comunidades y sobre todo a las comunidades de estatus mixto a través de sus campañas de activismo, mediante sus llamadas a la acción comunitaria y en su actividad congresional.

La particularidad de las localidades es que están definidas como espacios concretos de acción debajo del activismo institucional estatal y federal. En las ciudades, el cambio demográfico, social y cultural causado por las migraciones es más visible (Filomeno, 2017). Las urbes significan para los migrantes mayores oportunidades socioeconómicas y una mejor oferta de servicios sociales y políticas de acogida (De Graauw \& Vermeulen, 2016). También, como resultado de un mayor arraigo, hay mayores oportunidades para el activismo comunitario, mayores espacios de expresión para líderes étnicos y arenas políticas más permeables, es decir, en términos generales hay mayores posibilidades de éxito para el activismo comunitario, más foros para los líderes de opinión, y una vibrante vida cívica que hace más visibles los nichos políticos.

Los principales efectos del activismo de los migrantes en las ciudades se pueden apreciar claramente en las legislaciones santuario, en una mayor oferta de servicios de inmigración financiados con fondos públicos y en la creación de foros consultivos que los incluyen en el diseño político. Lo cierto es que el camino para llegar a esas conquistas políticas ha sido cuesta arriba y que el éxito depende del grado de movilización de las comunidades. 
INNOVACIÓN SOCIAL MIGRANTE Y EL MODELO DE INCIDENCIA POLÍTICA

Un problema de las comunidades migrantes es que sus necesidades específicas regularmente son diferentes a las de los ciudadanos promedio; por esta razón, frecuentemente no son participes de los proyectos de regeneración urbana en las zonas metropolitanas de asentamiento. Mucho menos cuando no están justamente representados en los órganos deliberativos y de diseño político, debido a los limitados derechos políticos que tienen en Estados Unidos. Las comunidades latinas de estatus mixto se caracterizan por localizarse en la periferia de los grandes centros urbanos y por la subinversión en sus vecindarios. Esto obliga a las comunidades a tener que organizarse para demandar la atención de sus representantes políticos, pero también a convertirse en fuentes de propuestas originales.

En los últimos años, los migrantes mexicanos en Estados Unidos están adoptando visiones pragmáticas sobre la política estadunidense; ahora reconocen que el restriccionismo y el conflicto son valores políticos inherentes de ese país y que la solución a sus problemas no acontecerá solamente mediante el incremento del voto latino. Al respecto, un líder migrante explica:

El problema con la política formal es que ellos [los políticos] pasan el tiempo solamente introduciendo iniciativas de ley para regularizar parcialmente a los inmigrantes, y de todos modos fallan en eso; pocas veces los políticos introducen reformas estructurales profundas que es lo que requiere este país (Entrevista, líder migrante, Chicago, marzo de 2017).

Los grupos de migrantes organizados consideran que esto sólo puede contrarrestarse a través del auto-empoderamiento y la organización comunitaria, que sólo así los inmigrantes van a tener el peso político para desafiar la segregación estructural y las desigualdades institucionales que persisten en sus lugares de asentamiento.

Los líderes migrantes consideran que ellos son quienes deben tomar las riendas de los proyectos y programas que involucran a sus comunidades. Un líder explica "nosotros sabemos mejor cuáles son las demandas de la comunidad, somos nosotros quienes convivimos todos los días con la raza y a quienes recurren cuando tienen problemas" (Entrevista, Santa Ana, junio de 2018). Otro líder argumenta que "todos quieren hablar por los migrantes... pero somos nosotros quienes tenemos que tomar las riendas de nuestra participación”(Entrevista, Chicago, mayo de 2017). 
La innovación social engloba a todas aquellas estrategias que buscan resolver los problemas comunitarios de forma creativa, apoyándose en personas con conocimiento práctico que haya resultado de la experiencia, mediante iniciativas sostenibles en el tiempo y que estén basadas en campañas de base (Gerometta et al., 2005; Mulgan et al., 2007). Con base en lo anterior podría pensarse que la innovación social es un nombre nuevo que se le da a viejas prácticas; sin embargo, su vigencia analítica para estudiar las prácticas de los colectivos migrantes de estatus mixto se basa en la mayor adopción de causas propias y en el posicionamiento como agentes de cambio en la vida social y cívica de sus comunidades, que ha estado floreciendo en las últimas décadas en EE.UU.

Este paradigma de la innovación social se caracteriza por percibir en los desafíos y amenazas, oportunidades para reconstruir, empoderar e incrementar la resiliencia de las comunidades (Grimm et al., 2013). Las comunidades de estatus mixto están enfrentando desafíos como el racismo, la retórica nativista, políticas de exclusión, desigualdades, desinversión y segregación; sin embargo, como resultado de tener que superar día con día y de la mano de la comunidad estas barreras, han surgido estrategias creativas e iniciativas importantes.

En general, las estrategias de emprendimiento social desarrolladas por los inmigrantes tienen como objetivo primario mejorar las condiciones de vida de las familias y también solucionar problemas de los vecindarios, los cuales tienen un alto carácter étnico. Sin embargo, la organización comunitaria, frecuentemente es la antesala de la participación política a mayor escala. Así, algunos proyectos de innovación social han escalado hasta convertirse en estrategias de incidencia política de las comunidades mixtas en el microcosmos extendido que representa la ciudad.

La incidencia política se relaciona directamente con las aspiraciones más altas de los inmigrantes que buscan influir sobre los procesos de toma de decisiones y diseño político de sus localidades a su favor. Los modelos de participación basados en la incidencia política buscan posicionar a los migrantes como colectivos propositivos y no solamente reactivos en la arena política local.

La incidencia política se refiere a campañas interseccionales gestadas en el seno de las organizaciones de inmigrantes, con el objetivo de vincular sus causas políticas a los proyectos de desarrollo urbano, a los programas públicos de la metrópoli, a las campañas de la sociedad civil local y a los asuntos de interés de las élites políticas locales. Estas campañas amplias implican un conjunto de iniciativas transversales que cubren múltiples asuntos, desde servicios de inmigración y ciudadanía, canalización de programas de acción afirmativa y temas de desarrollo comunitario para sus vecindarios, los cuales 
normalmente padecen de problemas de subinversión pública. Lo que diferencia a la incidencia política de otros modelos de participación desarrollados por las comunidades de migrantes mexicanos, es que implica una familiarización con la estructura de poder local para identificar quiénes son los responsables de la toma de decisiones y dónde hay canales de participación disponibles para sus grupos.

Organizaciones como WOLA (Washington Office on Latin American Affairs) y LULAC (League of United Latin American Citizens) han documentado y divulgan, como prácticas modelo, estas iniciativas de incidencia política desarrolladas por las comunidades migrantes mexicanas en Estados Unidos. El modelo de incidencia política que he podido documentar durante la observación participante con comunidades migrantes de California, Texas e Illinois consiste en varios pasos:

Primero, en la amplia agenda de las organizaciones de migrantes se deben identificar los problemas más urgentes y priorizar campos de acción, es decir, todos los migrantes saben que la reforma migratoria es una demanda compartida entre las comunidades latinas en Estados Unidos; sin embargo, más allá de la presión colectiva y el cabildeo con sus representantes, no hay mucho que las comunidades puedan hacer para resolver este tema pendiente en la agenda. Aunado a esto, la creciente diversificación en términos de clase, estatus migratorio y generación de las comunidades de origen migrante mexicano en Estados Unidos ha traído, a su vez, la diversificación de temas y prioridades. Esto ha dado como resultado una agenda política cada vez más amplia, que combina asuntos locales, estatales, federales y transnacionales. Entonces, esta fase inicial el modelo de incidencia política implica construir la agenda en común, consensuar prioridades y acordar acciones.

Después de la negociación de la agenda, durante una segunda etapa, los líderes deben identificar quién es el responsable dentro de la gobernanza local y quiénes podrían ayudarlos en el camino, convirtiéndose así en sus aliados políticos y en sus vínculos para ingresar a los corredores de poder de la ciudad. Este ejercicio implica un análisis y un cuestionamiento de las estructuras de poder locales. Una líder migrante explicaba "tenemos que preguntarnos: ¿quién es el oficial responsable de esto? y ¿quién puede ayudarnos con esta iniciativa?” (Notas de campo, Los Ángeles, junio de 2018). Aunque parece simple, es un gran avance: la mayoría de los ciudadanos no conocen a sus representantes y no hacen un análisis de sus aliados políticos. Este segundo paso ha sido vital para la adopción de causas políticas locales y para entender cómo, si bien hay temas que necesitan de activismo federal, hay otros asuntos a los que pueden hacer frente con 
apoyo de sus localidades, espacios donde además tienden a tener mayor peso político por su concentración demográfica.

El tercer paso consiste en forjar representaciones en la arena pública como agentes propositivos; para lograr esto, los líderes y las organizaciones migrantes promueven que sus ideas sean discutidas en los foros consultivos y que las buenas prácticas sean oficializadas para así poder tener el respaldo institucional y ser partícipes del presupuesto local. Los líderes migrantes constantemente explican a sus comunidades que la política se aprende con la práctica. Este proceso de promover iniciativas "desde abajo", basadas en la experiencia del trabajo comunitario, también ha tenido el efecto de vincular a los líderes migrantes con los políticos locales. A partir de esta relación de cercanía, más migrantes se vinculan con las maquinarias políticas y deciden iniciar campañas para representar a sus comunidades directamente en el gobierno local y estatal.

Los organizadores comunitarios latinos describen en sus foros que, para ellos, la incidencia política es el proceso de convertirse en un semillero de propuestas a través de “campañas amplias". Estrategias que logren potenciar en su beneficio a los programas institucionales existentes, pero a través de acción independiente o autonómica, es decir, estrategias que se conviertan en innovaciones comunitarias y que logren inspirar un cambio político “desde abajo”. Un líder de la organización Alianza Américas explica:

Las campañas amplias son aquellas que reúnen a ambas, a la identidad racial y a la identidad de clase que persisten en Estados Unidos... Nosotros creemos que como personas que pagan impuestos diariamente a nivel local, estatal y federal, necesitamos involucrarnos en procesos que nos permitan decirle a cualquier persona, sin importar si nació aquí o en algún otro lugar, que todos estamos en el mismo bote. Que estamos en la misma situación y que tal y como ellos están preocupados por asuntos de justicia económica, nosotros también lo estamos. Que es a educación, salud, seguridad pública, a las áreas a las que los recursos públicos deberían estar llegando y no a perseguir trabajadores inmigrantes (Entrevista, Chicago, mayo de 2017).

A partir de lo anterior, vemos cómo la incidencia política se refiere a los esfuerzos colectivos de inmigrantes organizados para influenciar la formulación e implementación de política pública mediante la presión colectiva basada en la persuasión política. En otras palabras, la incidencia política implica promover cambios basados en las experiencias concretas aprendidas de los problemas que los inmigrantes y las minorías étnicas enfrentan diariamente, pero de forma que puedan vincularlas con las demandas de otros sectores sociales locales o sumarlas a los proyectos de regeneración urbana existentes en la ciudad. Así, las organizaciones y líderes logran proyectar imágenes de actores 
propositivos, dispuestos no sólo a marchar por una reforma migratoria, sino a generar y escalar innovaciones comunitarias.

Los líderes latinos están convencidos de que es necesario: "ver en dónde hay esfuerzos exitosos para replicarlos, porque con los gobernantes hay una gran diferencia entre el discurso y la realidad” (Entrevista, Chicago, abril 2017). Como una líder comunitaria en California expresaba: "Tenemos que cuestionar a nuestros gobernantes, tenemos que ser críticos con nuestro sistema político, tenemos que demandar el reconocimiento de nuestros derechos, pero también tenemos que proponer iniciativas” (Rally, Santa Ana, junio de 2018). Precisamente este es el espíritu detrás de la innovación comunitaria y de sus posibilidades de incidencia política.

\section{BUENAS PRÁCTICAS CONSOLIDADAS}

Una vez explicado el modelo de innovación social y las estrategias de incidencia política de las comunidades migratorias mixtas en Estados Unidos, procederé a exponer algunas de las prácticas más innovadoras y exitosas documentadas durante el trabajo de campo. Primero se documentan las que se relacionan con la innovación comunitaria dirigida a mejorar el estatus social y económico de los colectivos; posteriormente se analizan las estrategias de incidencia política que buscan construir comunidades cívicas y políticas influyentes en los contextos metropolitanos de asentamiento.

Uno de los principales problemas que afecta a las comunidades inmigrantes y en particular a la identidad de sus enclaves étnicos es la gentrificación (Curran, 2017; Schuerman, 2019). Los latinos están enfrentando procesos de suburbanización que los empujan hacia áreas dormitorio alejadas de los centros urbanos, debido a la escasez de vivienda asequible o al repentino aumento de sus hipotecas por la valorización del barrio. El Este de Los Ángeles, La Villita en Chicago, East Harlem y El Segundo Barrio en Nueva York, y Logan en San Diego son comunidades simbólicas que históricamente han albergado a la economía étnica de los inmigrantes mexicanos, pero que también alojan a organizaciones culturales, comunitarias y políticas, de tal forma que son espacios de encuentro y socialización política para las comunidades migrantes de estatus mixto. Cuando los inmigrantes abandonan estos espacios urbanos y se dispersan por las áreas suburbanas, pierden cohesión social y también disminuye su influencia colectiva en la metrópoli. Ante esto, campañas denominadas "El barrio no se vende, se ama y se defiende" 
han proliferado en Estados Unidos. Destacan el Movimiento por Justicia del Barrio en Nueva York (Felix, 2017) y el movimiento de Pilsen en Chicago (Andries, 2017).

Uno de los argumentos centrales de los grupos antiinmigrantes es la falacia del hiperciudadano (Bleeden et al., 2010). Esta idea surge de los malentendidos de grupos nativistas de que ellos son los principales contribuyentes a la economía mediante sus impuestos sobre la renta, y que, por tanto, ellos son los dueños del gobierno. En esta situación, los hiperciudadanos tienen el derecho de excluir a los inmigrantes como sujetos de política pública. Es más, hay un creciente apoyo a políticas excluyentes como la de la regla de la carga pública. Esta regla implica que, si se sospecha que un inmigrante tiene posibilidades de convertirse en dependiente de ayudas gubernamentales, puede prohibírsele la entrada al país, y si ya está habitándolo, se debe proceder a su deportación.

Como respuesta a estos mitos, las organizaciones de inmigrantes han creado “campañas de justicia tributaria”. Su objetivo es concientizar a la población local sobre sus contribuciones sociales y económicas a las urbes de recepción. En estas campañas, los inmigrantes expresan, en los medios y en mítines, cómo en Estados Unidos los inmigrantes contribuyen con alrededor de \$12 000 millones dólares al sistema de seguridad social, y que muchos de ellos no pueden usar estos recursos por la falta de documentos (Notas de campo, reunión comunitaria, Los Ángeles, mayo 2017). Aunado a esto, en Chicago, las organizaciones de inmigrantes evidencian que $6.8 \%$ de los emprendedores de la ciudad son inmigrantes indocumentados y cómo "La 26 de La Villita" es la segunda calle comercial más importante de la ciudad, sólo después de la Milla Magnífica (Notas de campo, reunión comunitaria, Chicago, mayo 2017).

Otra estrategia de incidencia política de las comunidades de estatus mixto que se relaciona con aumentar la participación electoral del colectivo constituye lo que las organizaciones denominan "sacar el voto". Esta campaña involucra a todos los miembros del colectivo latino de estatus mixto, ya que es desarrollada por los líderes de las organizaciones étnicas para incorporar a los inmigrantes sin derechos políticos plenos a la movilización de votantes. Los líderes de migrantes observaron que los jóvenes latinos tienen bajas tasas de participación política y pocas intenciones para postularse por un cargo público. Por otro lado, algunos inmigrantes indocumentados quisieran tener el poder de participación electoral. Ante esta paradoja, los inmigrantes indocumentados trabajan como voluntarios para ofrecer información sobre cómo y en dónde registrarse para votar; asimismo, el día de la elección, facilitan transporte a adultos mayores y personas discapacitadas para que puedan acercarse a las urnas.

Clivajes. Revista de Ciencias Sociales (ISSN: 2395-9495), Año VIII, Núm. 15, enero-junio, 2021 Instituto de Investigaciones Histórico-Sociales, Universidad Veracruzana, México 
Agamben (1998) argumenta que: "la separación entre lo humanitario y lo político que actualmente estamos viviendo es la fase extrema de la ruptura entre los derechos humanos y los derechos civiles" (p. 169). Lo cierto es que el sistema migratorio estadunidense está estructurado para preservar el riesgo de deportabilidad de la mayoría de los trabajadores indocumentados. Robinson (2006) explica que: "los empleadores... quieren sostener una vasta base explotable de trabajo que exista bajo condiciones precarias, que no disfrute los mismos derechos civiles, políticos y laborales de los ciudadanos y que sea desechable a través de la deportación” (p. 84). En sintonía, una líder migrante explica que: "hay políticas de aún mantienen a nuestras comunidades indocumentadas porque los dos partidos políticos y el sistema opresivo se beneficia de nosotros" (Entrevista, Los Ángeles, mayo de 2018).

En el contexto de la presidencia de Donald Trump, su narrativa nativista y antiinmigrante, y su política de "tolerancia cero" a la inmigración, las comunidades de estatus mixto hicieron esfuerzos aún más exhaustivos de innovación social para mantener y maximizar su incidencia política. Hay dos campañas que particularmente destacaron durante esta época: en primer lugar, encontramos las campañas de "Conozca sus derechos" para inmigrantes indocumentados; en segundo lugar, tenemos a las campañas de ciudadanización.

Históricamente, los periodos de baja tolerancia política dificultan el planteamiento de opciones de regularización migratoria y desincentivan a los residentes permanentes de naturalizarse (Levin, 2013). Sin embargo, después de las elecciones presidenciales de 2016 en Estados Unidos, está pasando lo opuesto. El inminente riesgo de deportación de inmigrantes considerados no prioritarios y la posibilidad de incrementar el empoderamiento latino a través de expandir el peso electoral del colectivo, se percibieron como los mecanismos más viables para incrementar la resiliencia de las comunidades mexicanas de estatus mixto en este contexto de hostilidad.

En algunos vecindarios latinos, se organizaron grupos de voluntarios que constituían "Redes de Respuesta Rápida”. En estas campañas, voluntarios entrenados por las organizaciones alertaban sobre redadas y asistían a observar y videograbar las operaciones de ICE para evitar excesos policiacos y violaciones al debido proceso. En la misma dirección, otra innovación fue el “Check-involuntario con ICE”. Estas campañas aprovechaban que el Departamento de Seguridad Interior estadunidense argumentaba que ni los estudiantes, los padres de familia, ni las personas sin registro criminal eran una prioridad para las deportaciones. Así, miembros influyentes del colectivo asistían bajo buena voluntad y con el acompañamiento masivo de sus comunidades que ejercían presión 
colectiva, a reportar su presencia indocumentada en las oficinas de ICE para poder permanecer un año más en el país.

Adicionalmente a estas estrategias de innovación social, las comunidades crearon otras acciones de incidencia política orientadas a desarrollar las habilidades y competencias de los Latinos. Un ejemplo crucial han sido las campañas de “Conozca sus derechos”. Un líder migrante explica:

Es increíble como la comunidad todavía no sabe la enorme cantidad de derechos que tenemos en este país. Es increíble que se siguen equivocando en el procedimiento y que se auto condenan a deportación expedita. Luego, se acercan a nosotros pidiendo ayuda cuando ya nada se puede hacer (Discurso en taller de ciudadanía, Chicago, junio de 2017).

Las ligas cívicas, las organizaciones migrantes y las asociaciones étnicas han denunciado prácticas y violaciones de los oficiales de reforzamiento migratorio en las detenciones de ICE. Por ejemplo, encontramos que los oficiales de inmigración llegan a los lugares de trabajo o a los hogares sin una orden firmada por un juez, que ICE cuestiona por el estatus migratorio de una persona basándose en perfilamientos raciales y sin una orden de detención, así deportan a personas que originalmente no eran el objetivo. También piden a las personas firmar formatos de deportación voluntaria sin explicarles que es una acción irrevocable y sin proporcionar acceso a consejo legal.

Así, las campañas de "Conozca sus derechos" incluyen segmentos en radio, televisión, anuncios en las paradas de autobuses y hasta música, en las que se les explica a los inmigrantes sus derechos. Por ejemplo, se les enseña a distinguir entre los oficiales de ICE y la policía. Se les explica la diferencia entre los formatos que un oficial migratorio puede presentarles. Finalmente, se les enseñan sus derechos constitucionales como lo son el derecho a permanecer callado, el derecho a un abogado y la prerrogativa de no firmar nada sin consejo legal. Inclusive, se imprimieron millones de tarjetas bilingües que los inmigrantes podrían proporcionar a los oficiales migratorios en caso de ser detenidos. Con el escalamiento de las deportaciones, se crearon decenas de aplicaciones para smartphones en las que se reproducían los derechos de los inmigrantes $\mathrm{Y}$ en las que se avisaban a los familiares de la detención con sólo un botón.

Brubaker explica que en las sociedades contemporáneas "sólo para una minoría o parte marginal de la población, no hay duda o cuestionamiento sobre su membresía substantiva o su estatus ciudadano” (2010, p. 64). Para el resto de los individuos, su afiliación y su identidad son constantemente desafiadas y el ejercicio ciudadano se enfrenta a la hostilidad política.

Clivajes. Revista de Ciencias Sociales (ISSN: 2395-9495), Año VIII, Núm. 15, enero-junio, 2021 Instituto de Investigaciones Histórico-Sociales, Universidad Veracruzana, México 
Numerosos autores han estudiado el peso del voto Latino en Estados Unidos (Barreto, 2012; Stokes, 2003), estos estudios han predicho el crecimiento de Latinos elegibles como votantes, pero también han encontrado bajas tasas de naturalización entre residentes permanentes latinos. Unos cuantos meses antes de las elecciones de 2016, los líderes inmigrantes empezaron a discutir en sus foros internacionales sobre cómo incrementar el voto latino. Así, encontraron reportes que estaban demostrando que residentes permanentes mexicanos no se estaban naturalizando para obtener derechos civiles y políticos plenos en Estados Unidos (Gonzalez-Barrera, 2017). Sin embargo, el tiempo hacia la siguiente elección se estrechaba y los procesos de ciudadanía podrían tomar entre dos y cinco años.

Después de las alecciones, los reportes demostraron que cerca de 3.5 millones de migrantes latinos, la mayoría de ellos mexicanos, eran elegibles para obtener la ciudadanía en Estados Unidos (Lopez \& Stepler, 2016). Los líderes comunitarios hicieron sondeos en sus comunidades y encontraron que el alto costo de las tasas de naturalización (las cuáles equivalen aproximadamente a $\$ 880$ dólares), y actitudes como la desestimación de su nivel de inglés y el miedo a reprobar el examen cívico eran las principales barreras a la naturalización y que estas barreras podrían ser combatidas con el acompañamiento y apoyo comunitario.

Así es como surgieron los talleres de ciudadanía, que son eventos organizados por las asociaciones de inmigrantes, políticos proinmigrantes, consulados y otras organizaciones de la sociedad civil en los que se invita a los residentes a "dar el siguiente paso y naturalizarse”. Estos talleres de ciudadanía trabajan bajo modelos de clínicas legales que proveen acompañamiento logístico, consejería legal y también opciones de financiamiento para prevenir casos de fraude. Voluntarios de la comunidad participan ayudando a llevar los formatos, impartiendo clases de inglés como segunda lengua y practicando para el examen cívico. Inclusive se han logrado gestionar fondos para cubrir las tasas mediante donaciones de organizaciones filantrópicas.

Estos talleres de ciudadanía tendrán efectos a largo plazo sobre el incremento del voto latino debido a los tiempos de espera de los procesos de ciudadanía. Sin embargo, a corto y mediano plazo, constituyen escuelas políticas para las comunidades mexicanas de estatus mixto en las zonas metropolitanas de Estados Unidos sobre las posibilidades en la vida pública de sus comunidades de asentamiento. Estas estrategias han tenido efectos sobre la confianza política de las comunidades de estatus mixto y son catalizadores para la participación cívica, para la innovación social y para impulsar la incidencia política. 


\section{LA INNOVACIÓN COMUNITARIA DURANTE LA PANDEMIA DEL COVID-19}

La paulatina evolución comunitaria y la madurez política de las comunidades de estatus mixto de origen mexicano en Estados Unidos, que ha sido adquirida a partir de éxitos y fracasos, de experiencia y de experimentación, había permitido que la masa crítica guiando el activismo latino viera en 2020 un año clave para el empoderamiento del colectivo. Dos sucesos eran protagónicos en su activismo durante este periodo. Por un lado, el censo poblacional, en el que el principal desafío era lograr que se contara justamente a las comunidades de estatus mixto para que tuvieran una representación adecuada en los órganos políticos y para que gozaran de un presupuesto más equitativo. Por otro lado estaban las elecciones presidenciales de 2020, las cuales obligarían a reactivar y mejorar las estrategias de incidencia política como la movilización de votantes y las campañas de información para aumentar el peso electoral del colectivo.

Sin embargo, durante los primeros meses de 2020, la primera ola de la pandemia de Covid-19 azotó los principales centros urbanos de Estados Unidos. Esas zonas metropolitanas en donde habitan principalmente las comunidades de estatus migratorio mixto y cuyos vecindarios étnicos son los semilleros de innovación social e incidencia política de los latinos, se enfrentaron a confinamientos y otros desafíos en materia de salud, bienestar y economía.

Desde 2019, las organizaciones latinas en Estados Unidos habían lanzado campañas de información sobre el censo poblacional y la importancia de que se contara a todos en la comunidad de estatus migratorio mixto. Una líder de una organización en Texas explicaba en una transmisión de Facebook que: “ahorita las personas todavía no entienden la importancia de que todos y cada uno de nosotros quedemos registrados en el censo, piensan que por no ser ciudadanos no tienen que reportarse... El dinero que recibamos para nuestros proyectos comunitarios, para nuestras escuelas y facilidades públicas depende de que todos quedemos registrados" (Notas de campo, reunión comunitaria, Dallas, enero de 2020). Inclusive impulsaron litigios en cortes federales en contra de las intenciones del presidente Trump para cuestionar el estatus migratorio de la población en el Censo.

Las organizaciones de inmigrantes ofrecieron beneficios y servicios gratuitos para que las personas asistieran a llevar sus formularios del censo. Por ejemplo, una organización de Dallas ofrecía tamales y tacos gratuitos a todas las personas que se acercaran a consultar información y a llenar su boleta del censo. En el 2020, estas organizaciones mantuvieron tales campañas adaptándose a las medidas sanitarias y de

Clivajes. Revista de Ciencias Sociales (ISSN: 2395-9495), Año VIII, Núm. 15, enero-junio, 2021 Instituto de Investigaciones Histórico-Sociales, Universidad Veracruzana, México 
distanciamiento social, pero con el firme objetivo de que todos en su comunidad fueran contados.

El otro gran reto durante la pandemia fue la movilización de votantes durante las elecciones primarias y las elecciones presidenciales de 2020. Las comunidades migrantes de estatus mixto suelen habitar en ciudades que son sólidamente demócratas o republicanas, lo cual ocasiona que las elecciones primarias sean la arena importante en la que deben impulsar a los candidatos con agendas étnicas. Debido a diferentes sistemas de partido en sus lugares de origen y a un desconocimiento de cómo funciona la política estadunidense, los líderes de las organizaciones tienen que concientizar a sus comunidades de la importancia que tiene su participación en las elecciones primarias. Una líder de California convocaba en un Facebook Live:

¡Tiene tres formas diferentes de votar, pero hágalo, vote! Los latinos en California somos el bloque Latino más grande del país con 7.9 millones. En 2018 logramos incrementar el voto de nuestra gente en $200 \%$ y en estas elecciones vamos por más. Ayude principalmente a sus adultos mayores y a las personas que van a votar por primera vez para que se logre la meta (Discurso, Orange County, octubre de 2020).

Nuevamente y como en cada elección, las organizaciones comunitarias y étnicas pusieron en marcha una gran maquinaria para movilizar el voto latino, con la salvedad de que tuvieron que adaptar sus estrategias a las limitaciones causadas por la pandemia. Una de las principales preocupaciones de las organizaciones consistía en educar a sus comunidades sobre las reglas electorales locales, en particular sobre cómo votar sin exponer su salud, los plazos a considerar y sobre todo a tener confianza en que el voto anticipado o por correo podría hacer la diferencia. Para lograr esto, miles de voluntarios se involucraron en campañas puerta a puerta para sacar el voto en los vecindarios latinos. Posteriormente, estos voluntarios de las ligas urbanas que cuentan con más información hicieron viajes a las comunidades suburbanas "para organizar también esos espacios".

El resultado de esta campaña de movilización de votantes fue un incremento, en 30\%, del voto latino durante las elecciones de 2020 (Domínguez-Villegas et al., 2021). Los líderes destacan que los latinos de segunda generación fueron muy importantes en la movilización de votantes. Parece que los jóvenes latinos han heredado una imagen positiva del activismo comunitario, la innovación social y la incidencia política. Esto es clave en un contexto nacional en el que cada año un millón de latinos alcanzan la mayoría de edad (Domínguez-Villegas et al., 2021). Una líder migrante explica sobre éxito electoral: "No nos equivoquemos: los afroamericanos y los latinos ganamos la elección. Esto en una 
pandemia que ha afectado y ha matado desproporcionadamente a las comunidades negras y latinas" (Discurso, Los Ángeles, noviembre de 2020).

Según informes del Centro para el Control y Prevención de Enfermedades de Estados Unidos, existen tasas desproporcionadas de hospitalización y defunción entre comunidades latinas y afroamericanas con respecto a los blancos (Center for Disease Control and Prevention, 2021). Estos estudios revelan que los latinos fueron hospitalizados en promedio dos veces más que los blancos. El Center for American Progress reporta que, del total de empleos perdidos por la pandemia, un 23\% fue de latinos, y una vez iniciada la recuperación económica, el empleo latino sigue en déficit de 7.2\% en comparación con sus niveles prepandémicos (Zamarripa \& Roque, 2021). El 72\% de los hogares de las comunidades migrantes de estatus mixto reportaron que estaban teniendo problemas financieros severos por la pandemia, frente a sólo a un 36\% de los estadunidenses anglosajones que se encontraban en esa misma situación. Aunado a lo anterior, 6 de cada 10 adultos latinos encuestados reportan que han perdido sus trabajos o que les han reducido su sueldo como resultado de la pandemia (Robert Wood Johnson Foundation, 2020).

Varias circunstancias han confluido para que las minorías étnicas y raciales sean los colectivos más afectados por la pandemia del Covid19 en Estados Unidos. En primer lugar, tenemos que los Latinos fueron de los grupos que menos posibilidad tuvieron de trabajar de forma remota desde casa, debido a que primordialmente se desempeñan como trabajadores esenciales, en servicios de mantenimiento urbano, hostelería, limpia y cuidados, entre otras actividades que los mantuvieron más expuestos al virus. La segunda cuestión tiene que ver con la calidad de la vivienda: los latinos usualmente viven en barrios y en hogares con mayor hacinamiento. Finalmente, tenemos que considerar que las comunidades latinas de estatus migratorio mixto tienen menor acceso a los servicios de salud, incluyendo medicina preventiva, frente a precondiciones que afectan el desarrollo de casos severos o mortales de Covid-19 y también a medicina paliativa una vez adquirida la infección.

Precisamente la afectación desproporcionada de la pandemia por Covid-19 hacia los latinos ha ocasionado que las comunidades se organicen y movilicen para mitigar sus efectos sobre la salud y la economía de sus miembros. Los líderes comunitarios trabajaron para gestionar estímulos monetarios para que las personas indocumentadas también pudieran ser elegibles para un alivio económico, esto se logró en California y en Nueva York, pero también se canalizaron fondos en estados como Texas y Arizona, que usualmente apoyan menos a los inmigrantes indocumentados. 
Como anteriormente se señaló, la mayoría de los latinos viviendo en zonas urbanas de Estados Unidos, trabajan en el sector servicios. Muchos laboran como trabajadores esenciales de primera línea en los servicios de salud, de limpia, en centros comerciales y en la producción de alimentos. Aunado a esto, las comunidades de estatus migratorio mixto tienen una alta presencia en la industria de la hostelería y como comerciantes ambulantes. Estas actividades son las que mayormente enfrentaron cierres, restricciones de aforo y horario, recortes de personal y, en general, se vieron muy afectadas por la pandemia.

En este contexto, las comunidades de migrantes canalizaron fondos de sindicatos, cámaras de comercio y asociaciones filantrópicas para aquellos trabajadores migrantes que hubieran perdido sus trabajos como bartenders, meseros, vendedores ambulantes, personal de apoyo a conciertos, festivales y salas de cine, y músicos y artistas urbanos. Por ejemplo, las iniciativas "another round" y “CORE givers" ofrecieron estímulos de \$500 dólares al personal de hostelería con hijos menores de edad. En la misma línea, el Movimiento Cosecha logró recolectar donaciones que permitieron otorgar estímulos de $\$ 300$ dólares a trabajadores indocumentados agrícolas. También la Lee Foundation ofreció ayuda para pagar alimentos, el Urban Justice Center de Nueva York ofreció estímulos para los trabajadores ambulantes que hubieran dejado de laborar por causa de la pandemia. Asimismo, las organizaciones de migrantes canalizaron a sus miembros a iniciativas como "Rent Assistance" en los que asociaciones filantrópicas ayudaban con el pago de rentas e hipotecas a aquellas personas que no pudieran solventarlas por la pandemia.

Los líderes migrantes están concentrados en presionar a los gobiernos locales y estatales hacia lo que se llama "recuperación inclusiva y equitativa", argumentando que, si bien la pandemia afectó más desproporcionadamente a las comunidades de color, los planes de recuperación deberían fungir como grandes igualadores. Una activista de California explica "vamos a poder superar esto si nos mantenemos unidos como comunidad, no podemos dejar a los más débiles atrás” (Notas de campo, Los Ángeles, mayo de 2020). Parece que esta recuperación inclusiva se convertirá en el terreno principal para la incidencia política en el futuro cercano.

\section{DISCUSIÓN FINAL}

La hostilidad política federal que frecuentemente enfrentan los inmigrantes afecta sus conquistas locales, dificulta la articulación de sus demandas más allá de los ámbitos 
comunitarios inmediatos y complica la construcción de alianzas políticas con otros grupos vulnerables y con los insiders políticos. Riverstone (2017) explica que "cuando los gobiernos nacionales fallan para actuar sobre importantes asuntos sociales y económicos, los gobiernos locales entran en la brecha, emitiendo legislaciones que reflejan las condiciones, necesidades y deseos locales” (p. 418). En los contextos metropolitanos, la sociedad y el gobierno tiende a ver a los inmigrantes más como un beneficio para la vitalidad local que como una amenaza, por lo que se involucran en la experimentación política progresista en conjunto con colectivos migrantes que participan en la vida pública de la urbe.

Las comunidades latinas están en la búsqueda de lo que se denomina eficacia colectiva, una combinación de confianza mutua y voluntad grupal que les permita movilizarse bajo objetivos compartidos que promuevan la cohesión social dentro de la comunidad étnica (Browning et al., 2016). Estos procesos constituyen dinámicas botom-totop o "desde abajo" cuyo éxito depende de cómo se aprovechen los nichos políticos y de que la comunidad se mantenga políticamente activa. Las ganancias políticas de generar sus propias iniciativas bajo esquemas de experimentación cívica y política que se documentaron en este estudio se pueden resumir en un mayor profesionalismo en su activismo y más autonomía para implementar sus innovaciones comunitarias. Esto, a su vez, los posiciona como actores propositivos en la arena pública, llama la atención de sus representantes y también es la antesala de práctica política para que sus miembros decidan postularse para una oficina pública.

Para concluir, podemos sintetizar la innovación social y las estrategias de incidencia política de las comunidades de origen migrante como reflexiones colectivas con efectos tangibles, que permiten a los inmigrantes ser más conscientes de las posibilidades de participación en la vida cívica estadunidense $y$, además, contribuyen a construir comunidades políticas a través de la experimentación y la experiencia. Considerando el éxito de estas estrategias para mejorar el estatus socioeconómico del colectivo y los aprendizajes sobre política mediante la práctica misma, es muy probable que las comunidades de estatus mixto se sigan organizando para implementar proyectos de este tipo. En el futuro habrá que documentar el escalamiento de las innovaciones sociales y la incidencia política más allá del ámbito local y sobre todo su adopción en contextos más hostiles comparados con las grandes metrópolis objeto de este estudio.

El activismo y las iniciativas de las comunidades mexicanas de estatus mixto en las zonas metropolitanas de Estados Unidos pueden mitigar, pero no transformar abruptamente las condiciones estructurales de segregación y racismo que tienen que 
enfrentar. El antagonismo local-federal en la política migratoria, el desinterés de las élites políticas ante la falta de derechos políticos de colectivos que no garantizan un voto como retribución, los déficits presupuestarios y las desigualdades socioeconómicas intensificadas por la desinversión en sus comunidades, son cuestiones que no están directamente en las manos de las comunidades latinas. Probablemente, en el futuro, los latinos accederán a mayores posiciones de poder, con suerte representando íntegramente las demandas más contenciosas de sus comunidades étnicas; mientras tanto, el modelo de incidencia política que están desplegando es la estrategia más fructífera de empoderamiento social y de influencia política.

\section{REFERENCIAS}

Agamben, G. (1998). Homo Sacer: Sovereign Power and Bare Life. Standford University Press. ANDRIES, D. (2017). My neighborhood Pilsen. WTTW.

Barreto, M. A. (2012). Ethnic Cues: The Role of Shared Ethnicity in Latino Political Participation. University of Michigan Press.

Bleeden, D., Gottschalk-Druschke, C., \& Cintrón, R. (2010). Minutemen and the Subject of Democracy. En A. Pallares \& N. Flores-GonZÁlez (Eds.), Marcha! Latino Immigrants and the Immigrant Rights Movement (pp. 179-197). University of Illinois Press.

Browning, C. R., Dirlam, J., \& Boettner, B. (2016). From heterogeneity to concentration: Latino immigrant neighborhoods and collective efficacy perceptions in los angeles and chicago. Social Forces, 95(2), 779-807. https: / / doi.org/10.1093/sf/sow064

Brubaker, R. (2010). Migration, membership, and the Modern Nation-State : Internal and External Dimensions of the Politics of Belonging. Journal of Interdisciplinary History, 41(1), 61-78.

Center for Disease Control and Prevention. (2021). COVID-19 Racial and Ethnic Health Disparities. Recuperado de https: / /bit.ly/3Ib34Ts.

CôtÉ, I. (2013). Fieldwork in the era of social media: Opportunities and challenges. PS: Political Science \& Politics, 46(3), 615-619.

CRUz LERA, E. (2019). The spectrum of sanctuary cities in the United States: Contrasting the genesis and practices of proimmigrant local policies. Estudios Fronterizos, 20, 1-22. https: / / doi.org/10.21670/ref.1908029.

CURran, W. (2017). 'Mexicans love red' and other gentrification myths: Displacements 
and contestations in the gentrification of Pilsen, Chicago, USA. Urban Studies, September, 004209801773650. https: / / doi.org/10.1177/0042098017736503.

De Graauw, E., \& Vermeulen, F. (2016). Cities and the politics of immigrant integration: a comparison of Berlin, Amsterdam, New York City, and San Francisco. Journal of Ethnic and Migration Studies, 42(6), 989-1012. https: / / doi.org/10.1080/1369183X.2015.1126089.

Domínguez-Villegas, R., GonZalez, N., Gutierrez, A., Hernández, K., Herndon, M., Oaxaca, A. N. A., Rios, M., Roman, M., Rush, T. Y. E., \& Vera, D. (2021). Vote Choice of Latino Voters in the 2020 Presidential Election.

ENRIQUEZ, L. E. (2015). Multigenerational punishment: Shared experiences of undocumented immigration status within mixed-status families. Journal of Marriage and Family, 77(4), 939-953.

FElIX, D. (2017). The Fight to Save 'Our Beloved Barrio'. The Indypendent. Recuperado de https: / / bit.ly/3GGAcCc.

Filomeno, F. A. (2017). Theories of Local Immigration Policy. Palgrave Macmillan.

Gerometta, J., Häussermann, H., \& LongO, G. (2005). Social innovation and civil society in urban governance: Strategies for an inclusive city. Urban Studies, 42(11), 2007-2021. https: / / doi.org/10.1080/00420980500279851.

GonZalez-BarrerA, A. (2017). Mexican Lawful Immigrants Among the Least Likely to Become U.S. Citizens.

Grimm, R., Fox, C., Baines, S., \& Albertson, K. (2013). Social innovation, an answer to contemporary societal challenges? Locating the concept in theory and practice. Innovation: The European Journal of Social Science Research, 26(4), 436-455.

Kosinski, M., Matz, S. C., Gosling, S. D., Popov, V., \& Stillwell, D. (2015). Facebook as a research tool for the social sciences: Opportunities, challenges, ethical considerations, and practical guidelines. American Psychologist, 70(6), 543.

Krogstad, J. M., Noe-Bustamante, L., \& Flores, A. (2019). Voter turnout rose in 2018 across racial, ethnic groups | Pew Research Center. https://pewrsr.ch/33FDUOfLevin, I. (2013). Political Inclusion of Latino Immigrants: Becoming a Citizen and Political Participation. American Politics Research, 41(4), 535-568. Recuperado de https: / / doi.org/10.1177/1532673X12461438.

LOPEZ, M. H., \& STEPLER, R. (2016). Latinos in the 2016 Election: Illinois.

Mangual FigueroA, A. (2012). "I have papers so I can go anywhere!”: Everyday talk about citizenship in a mixed-status Mexican family. Journal of Language, Identity \& Education, 11(5), 291-311. 
Martiniello, M., \& LAfleuR, J. (2008). Towards a transatlantic dialogue in the study of immigrant political transnationalism. Ethnic and Racial Studies, 31(4), 645-663. https: / / doi.org/10.1080/01419870701784471.

Mollenkopf, J. H., \& Sonenshein, R. (2009). The New Urban Politics of Integration: A View from the Gateway Cities. En Bringing outsiders in: Trasatlantic perspectives on immigrant political incorporation. (pp. 74-92). Cornell University Press.

Mulgan, G., Simon Tucker, Ali, R., \& Sanders, B. (2007). Social Innovation: What it is , why it matters and what we can do about it. The Young Foundation.

PASSEl, J. S., \& COHN, D. (2017). 20 metro areas are home to six-in-ten unauthorized immigrants in U.S. En Pew Research Center. Recuperad de https: / / pewrsr.ch/3nyzqiW.

Price, M., \& Chacko, E. (2016). Migrants' Inclusion in Cities Innovative Urban Policies and Practices.

Riverstone-Newell, L. (2017). The Rise of State Preemption Laws in Response to Local Policy Innovation. Publius: The Journal of Federalism, 47(3), 403-425. https://doi.org/10.1093/publius/pjx037.

Robert Wood Johnson Foundation. (2020). Poll: Half of Households in the Four Largest U.S. Cities Report Serious Financial Problems Including Depleted Savings, and Trouble Paying Bills or Affording Medical Care. Recuperado de https: / / rwjf.ws/3tAHqnp.

Robinson, W. I. (2006). "Aqui estamos y no nos vamos!” Global capital and immigrant rights. Race and Class, 48(2), 77-91. https:/ / doi.org/10.1177/0306396806069525.

SASSEN, S. (1990). The mobility of labor and capital: A study in international investment and labor flow. Cambridge University Press.

SCHAEFFER, K. (2019). In a rising number of U.S. counties, Hispanic and black Americans are the majority. Pew Research Center. Recuperado de https://pewrsr.ch/3tIfezO.

SCHUERMAn, M. L. (2019). Newcomers: Gentrification and its Discontents. University of Chicago Press.

Stokes, A. K. (2003). Latino Group Conciousness and Political Participation. American Politics Research, 31(4). https: / / doi.org/10.1177/1532673X03252531.

Varsanyi, M. W., Lewis, P. G., Provine, D. M., \& Decker, S. (2011). A Multilayered Jurisdictional Patchwork: Immigration Federalism in the United States. Law and Policy, 34(2), 138-158. https://doi.org/10.1111/j.1467-9930.2011.00356.x.

ZAMARRIPA, R., \& ROQUE, L. (2021). Latinos Face Disproportionate Health and Economic Impacts From COVID-19. Recuperado de https: / / ampr.gs/33KCwcU. 